\title{
AN ANALOG OF NEIFELD'S CONNECTION INDUCED ON THE SPACE OF CENTRED PLANES
}

\author{
OLGA BELOVA
}

Received 26 March, 2018

\begin{abstract}
The paper concerns to investigations in the field of differential geometry. It is realized by a method of prolongations and scopes of G.F. Laptev which generalizes a moving frame method and Cartan's exterior forms method and bases on calculation of exterior differential forms. The space $\Pi$ of centred $m$-planes is considered in projective space $P_{n}$. Principal fiber bundle is arised above $\Pi$. An analog of Neifeld's connection is given in this principal fibering. It is proved that the analog of the Norden normalization of the space of centred planes induces Neifeld's connection. The torsion of Neifeld's connection analog is introduced. It is shown, that this object is a tensor.
\end{abstract}

2010 Mathematics Subject Classification: 53A20; 53B25; 53B15

Keywords: projective space, space of centred planes, principal fiber bundle, Neifeld's connection

\section{INTRODUCTION}

The object of research of this paper is an analog of Neifeld's connection on the space of centred planes.

We consider the space of centred planes as space of all $m$-dimensional centred planes in $n$-dimensional projective space. The non-classical analytical method is applied in this paper. It has advantage at allocation of subgroups and factor groups of a projective group. The connections in the fiberings associated with Grassmann manifold and the space of centred planes were investigated in [1] by such method. We show that an analog of Neifeld's connection is induced on the space of centred planes. By methods of tensor analysis E.G. Neifeld considered two dual linear connections (compare [3]), associated with normalized Grassmann manifold.

We put projective space $P_{n}$ to the moving frame $\left\{A, A_{I}\right\}(I, J, K, \ldots=\overline{1, n})$ with the derivation formulae

$$
d A=\theta A+\omega^{I} A_{I}, \quad d A_{I}=\theta A_{I}+\omega_{I}^{J} A_{J}+\omega_{I} A .
$$

The Pfaffian forms $\omega^{I}, \omega_{J}^{I}, \omega_{I}$ satisfy the Cartan structure equations of the projective group $G P(n)$ (see, eg., [5]):

$$
D \omega^{I}=\omega^{J} \wedge \omega_{J}^{I}, \quad D \omega_{I}=\omega_{I}^{J} \wedge \omega_{J},
$$

(c) 2018 Miskolc University Press 


$$
D \omega_{J}^{I}=\omega_{J}^{K} \wedge \omega_{K}^{I}+\delta_{J}^{I} \omega_{K} \wedge \omega^{K}+\omega_{J} \wedge \omega^{I} .
$$

\section{THE SPACE OF CENTRED PLANES}

The space $\Pi$ [1] of all centred $m$-dimensional planes $L_{m}^{*}$ is considered in $P_{n}$. Let's produce a specialization of the moving frame $\left\{A, A_{a}, A_{\alpha}\right\}(a, \cdots=\overline{1, m} ; \quad \alpha, \cdots=$ $\overline{m+1, n}$ ) putting the top $A$ in the centre of $m$-dimensional plane $L_{m}$ and putting the tops $A_{a}$ on the plane $L_{m}$.

From the derivation formulae (1.1) it follows that the equations $\omega^{\alpha}=0, \omega^{a}=0$, $\omega_{a}^{\alpha}=0$ are stationarity conditions of the centred planes $L_{m}^{*}$, i.e. forms $\omega^{\alpha}, \omega^{a}$, $\omega_{a}^{\alpha}$ are principal forms for the space $\Pi$. The basic forms of the space $\Pi$ satisfy the equations following from the structure Cartan equations (1.2)

where

$$
\begin{gathered}
D \omega^{\alpha}=\omega^{a} \wedge \Omega_{a}^{\alpha}+\omega^{\beta} \wedge \Omega_{\beta}^{\alpha}, \\
D \omega^{a}=\omega^{b} \wedge \Omega_{b}^{a}+\omega^{\alpha} \wedge \Omega_{\alpha}^{a}, \\
D \omega_{a}^{\alpha}=\omega_{b}^{\beta} \wedge \Omega_{\beta a}^{\alpha b}+\omega^{\alpha} \wedge \Omega_{a},
\end{gathered}
$$

$$
\Omega_{\beta}^{\alpha}=\omega_{\beta}^{\alpha}, \quad \Omega_{b}^{a}=\omega_{b}^{a}, \quad \Omega_{\alpha}^{a}=\omega_{\alpha}^{a}, \quad \Omega_{a}=-\omega_{a}, \quad \Omega_{\beta a}^{\alpha b}=\delta_{a}^{b} \Omega_{\beta}^{\alpha}-\delta_{\beta}^{\alpha} \Omega_{a}^{b} .
$$

Remark 1. The forms $\Omega_{a}^{\alpha}=\omega_{a}^{\alpha}$ are the basic-fibre forms.

Differentiating the forms (2.2) we obtain

where

$$
\begin{gathered}
D \Omega_{b}^{a}=\Omega_{b}^{c} \wedge \Omega_{c}^{a}+\omega^{\alpha} \wedge \Omega_{b \alpha}^{a}+\omega^{c} \wedge \Omega_{b c}^{a}+\omega_{b}^{\alpha} \wedge \Omega_{\alpha}^{a}, \\
D \Omega_{\beta}^{\alpha}=\Omega_{\beta}^{\gamma} \wedge \Omega_{\gamma}^{\alpha}+\omega^{\gamma} \wedge \Omega_{\beta \gamma}^{\alpha}+\omega^{a} \wedge \Omega_{\beta a}^{\alpha}-\omega_{a}^{\alpha} \wedge \Omega_{\beta}^{a}, \\
D \Omega_{\alpha}^{a}=\Omega_{b \alpha}^{\beta a} \wedge \Omega_{\beta}^{b}+\omega^{a} \wedge \Omega_{\alpha}, \\
D \Omega_{a}=\Omega_{a}^{b} \wedge \Omega_{b}+\omega_{a}^{\alpha} \wedge \Omega_{\alpha},
\end{gathered}
$$

$$
\begin{gathered}
\Omega_{\beta \gamma}^{\alpha}=-\delta_{\beta}^{\alpha} \omega_{\gamma}-\delta_{\gamma}^{\alpha} \omega_{\beta}, \quad \Omega_{\beta a}^{\alpha}=-\delta_{\beta}^{\alpha} \omega_{a}, \\
\Omega_{b c}^{a}=-\delta_{b}^{a} \omega_{c}-\delta_{c}^{a} \omega_{b}, \quad \Omega_{b \alpha}^{a}=-\delta_{b}^{a} \omega_{\alpha}, \quad \Omega_{\alpha}=-\omega_{\alpha} .
\end{gathered}
$$

The principal fiber bundle $\mathfrak{L}(\Pi)$ is constructed over the space $\Pi$ of centred planes and the Lie group $\mathfrak{L}$ is the typical fiber. This group acts in the tangent space [2] to the space $\Pi$.

Theorem 1. The principal fiber bundle $\mathfrak{L}(\Pi)$ contains the following factorfiberings:

(1) factorfibering of linear frames belonging to the centred plane $L_{m}^{*}$ which typical fiber is linear factorgroup acting in a bunch of lines belonging to a plane $L_{m}^{*}$, with the structure equations (2.1) and (2.3);

(2) factorfibering of normal linear frames with the structure equations (2.1) and (2.4); 
(3) factorfibering of coaffine frames belonging to the plane $L_{m}^{*}$ which typical fiber is coaffine factorgroup acting in the centred plane with the structure equations (2.1), (2.3) and (2.6);

(4) maximal affine factorfibering which typical fiber is factorgroup acting in a bunch of lines with centre A; the factorfibering has structure equations (2.1) and $(2.3-2.5)$.

\section{AN ANALOG OF NEIFELD'S CONNECTION}

In the principal fiber bundle we set an analog of Neifeld's connection [3] by the Laptev - Lumiste way.

Entering new forms

$\tilde{\Omega}_{b}^{a}=\Omega_{b}^{a}-\Gamma_{b \alpha}^{a} \omega^{\alpha}-\Gamma_{b c}^{a} \omega^{c}-\Gamma_{b \alpha}^{a c} \omega_{c}^{\alpha}, \quad \tilde{\Omega}_{\beta}^{\alpha}=\Omega_{\beta}^{\alpha}-\Gamma_{\beta \gamma}^{\alpha} \omega^{\gamma}-\Gamma_{\beta a}^{\alpha} \omega^{a}-\Gamma_{\beta \gamma}^{\alpha a} \omega_{a}^{\gamma}$,

$\tilde{\Omega}_{\alpha}^{a}=\Omega_{\alpha}^{a}-\Gamma_{\alpha \beta}^{a} \omega^{\beta}-\Gamma_{\alpha b}^{a} \omega^{b}-\Gamma_{\alpha \beta}^{a b} \omega_{b}^{\beta}, \quad \tilde{\Omega}_{a}=\Omega_{a}-\Gamma_{a \alpha} \omega^{\alpha}-\Gamma_{a b} \omega^{b}-L_{a \alpha}^{b} \omega_{b}^{\alpha}$

and finding their exterior differentials we get that connection in the principal fiber bundle $\mathfrak{L}(\Pi)$ is set with the help of a field of connection object

$$
\Gamma=\left\{\Gamma_{b \alpha}^{a}, \Gamma_{b c}^{a}, \Gamma_{b \alpha}^{a c}, \Gamma_{\beta \gamma}^{\alpha}, \Gamma_{\beta a}^{\alpha}, \Gamma_{\beta \gamma}^{\alpha a}, \Gamma_{\alpha \beta}^{a}, \Gamma_{\alpha b}^{a}, \Gamma_{\alpha \beta}^{a b}, \Gamma_{a \alpha}, \Gamma_{a b}, L_{a \alpha}^{b}\right\}
$$

on the base $\Pi$. We found differential equations of the connection object components:

$$
\begin{gathered}
\Delta \Gamma_{b \alpha}^{a}-\Gamma_{b c}^{a} \Omega_{\alpha}^{c}-\Gamma_{b \alpha}^{a c} \Omega_{c}+\delta_{b}^{a} \Omega_{\alpha}=\left.\Gamma_{b \alpha}^{a}\right|_{\beta} \omega^{\beta}+\left.\Gamma_{b \alpha}^{a}\right|_{c} \omega^{c}+\left.\Gamma_{b \alpha}^{a}\right|_{\beta} ^{c} \omega_{c}^{\beta}, \\
\Delta \Gamma_{b c}^{a}+\delta_{b}^{a} \Omega_{c}+\delta_{c}^{a} \Omega_{b}=\left.\Gamma_{b c}^{a}\right|_{\alpha} \omega^{\alpha}+\left.\Gamma_{b c}^{a}\right|_{e} \omega^{e}+\left.\Gamma_{b c}^{a}\right|_{\alpha} ^{e} \omega_{e}^{\alpha}, \\
\Delta \Gamma_{b \alpha}^{a c}+\delta_{b}^{c} \Omega_{\alpha}^{a}=\left.\Gamma_{b \alpha}^{a c}\right|_{\beta} \omega^{\beta}+\left.\Gamma_{b \alpha}^{a c}\right|_{e} \omega^{e}+\left.\Gamma_{b \alpha}^{a c}\right|_{\beta} ^{e} \omega_{e}^{\beta}, \\
\Delta \Gamma_{\beta \gamma}^{\alpha}-\Gamma_{\beta a}^{\alpha} \Omega_{\gamma}^{a}-\Gamma_{\beta \gamma}^{\alpha a} \Omega_{a}+\Omega_{\beta \gamma}^{\alpha}=\left.\Gamma_{\beta \gamma}^{\alpha}\right|_{\mu} \omega^{\mu}+\left.\Gamma_{\beta \gamma}^{\alpha}\right|_{a} \omega^{a}+\left.\Gamma_{\beta \gamma}^{\alpha}\right|_{\mu} ^{a} \omega_{a}^{\mu}, \\
\Delta \Gamma_{\beta a}^{\alpha}+\delta_{\beta}^{\alpha} \Omega_{a}=\left.\Gamma_{\beta a}^{\alpha}\right|_{\gamma} \omega^{\gamma}+\left.\Gamma_{\beta a}^{\alpha}\right|_{b} \omega^{b}+\left.\Gamma_{\beta a}^{\alpha}\right|_{\gamma} ^{b} \omega_{b}^{\gamma}, \\
\Delta \Gamma_{\beta \gamma}^{\alpha a}-\delta_{\gamma}^{\alpha} \Omega_{\beta}^{a}=\left.\Gamma_{\beta \gamma}^{\alpha a}\right|_{\mu} \omega^{\mu}+\left.\Gamma_{\beta \gamma}^{\alpha a}\right|_{b} \omega^{b}+\left.\Gamma_{\beta \gamma}^{\alpha a}\right|_{\mu} ^{b} \omega_{b}^{\mu}, \\
\Delta \Gamma_{\alpha \beta}^{a}-\Gamma_{\alpha b}^{a} \Omega_{\beta}^{b}-\Gamma_{\alpha \beta}^{a b} \Omega_{b}-\Gamma_{b \beta}^{a} \Omega_{\alpha}^{b}+\Gamma_{\alpha \beta}^{\gamma} \Omega_{\gamma}^{a}=\left.\Gamma_{\alpha \beta}^{a}\right|_{\gamma} \omega^{\gamma}+\left.\Gamma_{\alpha \beta}^{a}\right|_{b} \omega^{b}+\left.\Gamma_{\alpha \beta}^{a}\right|_{\gamma} ^{b} \omega_{b}^{\gamma}, \\
\Delta \Gamma_{\alpha b}^{a}-\Gamma_{c b}^{a} \Omega_{\alpha}^{c}+\Gamma_{\alpha b}^{\beta} \Omega_{\beta}^{a}+\delta_{b}^{a} \Omega_{\alpha}=\left.\Gamma_{\alpha b}^{a}\right|_{\beta} \omega^{\beta}+\left.\Gamma_{\alpha b}^{a}\right|_{c} \omega^{c}+\left.\Gamma_{\alpha b}^{a}\right|_{\beta} ^{c} \omega_{c}^{\beta}, \\
\Delta \Gamma_{\alpha \beta}^{a b}+\Gamma_{\alpha \beta}^{\gamma b} \Omega_{\gamma}^{a}-\Gamma_{c \beta}^{a b} \Omega_{\alpha}^{c}=\left.\Gamma_{\alpha \beta}^{a b}\right|_{\gamma} \omega^{\gamma}+\left.\Gamma_{\alpha \beta}^{a b}\right|_{c} \omega^{c}+\left.\Gamma_{\alpha \beta}^{a b}\right|_{\gamma} ^{c} \omega_{c}^{\gamma}, \\
\Delta \Gamma_{a \alpha}-\Gamma_{a b} \Omega_{\alpha}^{b}+\left(\Gamma_{a \alpha}^{b}-L_{a \alpha}^{b}\right) \Omega_{b}=\left.\Gamma_{a \alpha}\right|_{\beta} \omega^{\beta}+\left.\Gamma_{a \alpha}\right|_{b} \omega^{b}+\left.\Gamma_{a \alpha}\right|_{\beta} ^{b} \omega_{b}^{\beta}, \\
\Delta \Gamma_{a b}+\Gamma_{a b}^{c} \Omega_{c}=\left.\Gamma_{a b}\right|_{\alpha} \omega^{\alpha}+\left.\Gamma_{a b}\right|_{c} \omega^{c}+\left.\Gamma_{a b}\right|_{\alpha} ^{c} \omega_{c}^{\alpha}, \\
\Delta L_{a \alpha}^{b}+\Gamma_{a \alpha}^{c b} \Omega_{c}+\delta_{a}^{b} \Omega_{\alpha}=\left.L_{a \alpha}^{b}\right|_{\beta} \omega^{\beta}+\left.L_{a \alpha}^{b}\right|_{c} \omega^{c}+\left.L_{a \alpha}^{b}\right|_{\beta} ^{c} \omega_{c}^{\beta} .
\end{gathered}
$$

There are Pfaffian derivatives in the right side of the equations (3.1). 
Theorem 2. The object of group connection $\Gamma$ contains four simple geometric subobjects $\Gamma_{1}=\left\{\Gamma_{b \alpha}^{a c}, \Gamma_{b c}^{a}, \Gamma_{b \alpha}^{a}\right\}, \Gamma_{2}=\left\{\Gamma_{\beta \gamma}^{\alpha a}, \Gamma_{\beta a}^{\alpha}, \Gamma_{\beta \gamma}^{\alpha}\right\}, \Gamma_{3}=\left\{\Gamma_{1}, \Gamma_{a \alpha}, \Gamma_{a b}, \notin_{a \alpha}^{b}\right\}$, $\Gamma_{4}=\left\{\Gamma_{1}, \Gamma_{2}, \Gamma_{\alpha b}^{a}, \Gamma_{\alpha \beta}^{a b}, \Gamma_{\alpha \beta}^{a}\right\}$ giving the connection in the corresponding factorfiberings.

We realize an analogue of the strong Norden normalization [4] of the manifold by fields of the following geometrical images: $(n-m-1)$-dimensional plane $P_{n-m-1}$, not having the common points with the plane $L_{m}^{*}$, and $(m-1)$-dimensional plane $P_{m-1}$, belonging to the plane $L_{m}^{*}$ and not passing through its centre $A$.

We define the planes $P_{n-m-1}$ and $P_{m-1}$ by the points, respectively

$$
B_{\alpha}=A_{\alpha}+\lambda_{\alpha}^{a} A_{a}+\lambda_{\alpha} A, \quad B_{a}=A_{a}+\lambda_{a} A .
$$

Demanding a relative invariancy of the clothing planes we obtain the differential equations for the components of clothing geometrical object

$$
\Delta \lambda_{\alpha}^{a}+\Omega_{\alpha}^{a} \equiv 0, \quad \Delta \lambda_{\alpha}-\lambda_{\alpha}^{a} \Omega_{a}-\omega_{\alpha} \equiv 0, \quad \Delta \lambda_{a}-\Omega_{a} \equiv 0 .
$$

The analog of the strong Norden normalization [4] of the spaces $\Pi$ allows to cover the components of the connection object $\Gamma$

$$
\begin{gathered}
\Gamma_{b \alpha}^{a c}=\delta_{b}^{c} \lambda_{\alpha}^{a}, \quad \Gamma_{b c}^{a}=-\delta_{b}^{a} \lambda_{c}-\delta_{c}^{a} \lambda_{b}, \quad \Gamma_{b \alpha}^{a}=-\delta_{b}^{a} \mu_{\alpha}+\lambda_{\alpha}^{a} \lambda_{b}, \\
\Gamma_{\beta \gamma}^{\alpha a}=-\delta_{\gamma}^{\alpha} \lambda_{\beta}^{a}, \quad \Gamma_{\beta a}^{\alpha}=-\delta_{\beta}^{\alpha} \lambda_{a}, \quad \Gamma_{\beta \gamma}^{\alpha}=-\delta_{\gamma}^{\alpha} \lambda_{\beta}-\delta_{\beta}^{\alpha} \mu_{\gamma}, \\
\Gamma_{\alpha \beta}^{a b}=-\lambda_{\alpha}^{b} \lambda_{\beta}^{a}, \quad \Gamma_{\alpha b}^{a}=-\delta_{b}^{a} \mu_{\alpha}, \quad \Gamma_{\alpha \beta}^{a}=-\lambda_{b} \lambda_{\alpha}^{b} \lambda_{\beta}^{a}, \\
L_{a \alpha}^{b}=-\delta_{a}^{b} \lambda_{\alpha}, \quad \Gamma_{a b}=\lambda_{a} \lambda_{b}, \quad \Gamma_{a \alpha}=-\lambda_{a} \lambda_{b} \lambda_{\alpha}^{b},
\end{gathered}
$$

where $\mu_{\alpha}=\lambda_{\alpha}-\lambda_{\alpha}^{a} \lambda_{a}$.

The functions (3.3) by virtue of the comparisons (3.2) satisfy the differential equations (3.1) for the components of the connection object $\Gamma$.

Thus, we have

Theorem 3. The analog of the strong Norden normalization of the space $\Pi$ induces analog of Neifeld's connection in the associated fibering $\mathfrak{L}(\Pi)$.

\section{A TORSION OF NEIFELD'S CONNECTION ANALOG ON THE SPACE OF CENTRED PLANES}

Putting into the structure equations (2.1) of the basic forms $\omega^{\alpha}, \omega^{a}$ and $\omega_{a}^{\alpha}$ of the space $\Pi$ the connection forms $\tilde{\Omega}_{b}^{a}, \tilde{\Omega}_{\beta}^{\alpha}, \tilde{\Omega}_{\alpha}^{a}, \tilde{\Omega}_{a}$ we obtain the equations:

$$
\begin{aligned}
& D \omega^{\alpha}=\omega^{\beta} \wedge \tilde{\Omega}_{\beta}^{\alpha}+\underline{\omega^{a} \wedge \omega_{a}^{\alpha}}+S_{\beta \gamma}^{\alpha} \omega^{\beta} \wedge \omega^{\gamma}+S_{\beta a}^{\alpha} \omega^{\beta} \wedge \omega^{a}+S_{\beta \gamma}^{\alpha a} \omega^{\beta} \wedge \omega_{a}^{\gamma}, \\
& D \omega_{a}^{\alpha}=\omega_{b}^{\beta} \wedge\left(\delta_{a}^{b} \tilde{\Omega}_{\beta}^{\alpha}-\delta_{\beta}^{\alpha} \tilde{\Omega}_{a}^{b}\right)+\omega^{\alpha} \wedge \tilde{\Omega}_{a}+S_{a \beta \gamma}^{\alpha} \omega^{\beta} \wedge \omega^{\gamma}+S_{a \beta b}^{\alpha} \omega^{\beta} \wedge \omega^{b}+ \\
& S_{a \beta \gamma}^{\alpha b} \omega^{\beta} \wedge \omega_{b}^{\gamma}+S_{a \beta c}^{\alpha b} \omega^{c} \wedge \omega_{b}^{\beta}+S_{a \beta \gamma}^{\alpha b c} \omega_{b}^{\beta} \wedge \omega_{c}^{\gamma}, \\
& D \omega^{a}=\omega^{b} \wedge \tilde{\Omega}_{b}^{a}+\omega^{\alpha} \wedge \tilde{\Omega}_{\alpha}^{a}+S_{\alpha \beta}^{a} \omega^{\alpha} \wedge \omega^{\beta}+S_{\alpha b}^{a} \omega^{\alpha} \wedge \omega^{b}+S_{\alpha \beta}^{a b} \omega^{\alpha} \wedge \omega_{b}^{\beta}+
\end{aligned}
$$




$$
S_{b c}^{a} \omega^{b} \wedge \omega^{c}+S_{b \alpha}^{a c} \omega^{b} \wedge \omega_{c}^{\alpha}
$$

where

$$
\begin{gathered}
S_{\beta \gamma}^{\alpha}=\Gamma_{[\beta \gamma]}^{\alpha}, \quad S_{\beta a}^{\alpha}=\Gamma_{\beta a}^{\alpha}, \quad S_{\beta \gamma}^{\alpha a}=\Gamma_{\beta \gamma}^{\alpha a}, \\
S_{a \beta \gamma}^{\alpha}=\delta_{[\beta}^{\alpha} \Gamma_{a \gamma]}, \quad S_{a \beta b}^{\alpha}=\delta_{\beta}^{\alpha} \Gamma_{a b}, \quad S_{a \beta \gamma}^{\alpha b}=\delta_{\beta}^{\alpha} L_{a \gamma}^{b}+\delta_{\gamma}^{\alpha} \Gamma_{a \beta}^{b}-\delta_{a}^{b} \Gamma_{\gamma \beta}^{\alpha}, \\
\left.\left.S_{a \beta c}^{\alpha b}=\delta_{\beta}^{\alpha} \Gamma_{a c}^{b}-\delta_{a}^{b} \Gamma_{\beta c}^{\alpha}, \quad S_{a \beta c}^{\alpha b c}=\delta_{a}^{\lceil b} \Gamma_{\lfloor\beta \gamma}^{\alpha c}\right]-\delta_{\lfloor\beta}^{\alpha} \Gamma_{a \gamma}^{\lceil b c}\right], \\
S_{\alpha \beta}^{a}=\Gamma_{[\alpha \beta]}^{a}, \quad S_{\alpha b}^{a}=\Gamma_{\alpha b}^{a}-\Gamma_{b \alpha}^{a}, \quad S_{\alpha \beta}^{a b}=\Gamma_{\alpha \beta}^{a b}, \quad S_{b c}^{a}=\Gamma_{[b c]}^{a}, \quad S_{b \alpha}^{a c}=\Gamma_{b \alpha}^{a c} .
\end{gathered}
$$

Here the square brackets mean an alternation with the last indices and pairs of indices.

We represent the underlined term in formula (4.1) as

$$
\omega^{a} \wedge \omega_{a}^{\alpha}=\delta_{\beta}^{\alpha} \delta_{b}^{a} \omega^{b} \wedge \omega_{a}^{\beta} .
$$

Then the equation (4.1) takes the form

$$
D \omega^{\alpha}=\omega^{\beta} \wedge \tilde{\Omega}_{\beta}^{\alpha}+S_{\beta b}^{\alpha a} \omega^{b} \wedge \omega_{a}^{\beta}+S_{\beta \gamma}^{\alpha} \omega^{\beta} \wedge \omega^{\gamma}+S_{\beta a}^{\alpha} \omega^{\beta} \wedge \omega^{a}+S_{\beta \gamma}^{\alpha a} \omega^{\beta} \wedge \omega_{a}^{\gamma},
$$

where we use the new components $S_{\beta b}^{\alpha a}=\delta_{\beta}^{\alpha} \delta_{b}^{a}$ of the torsion $S$.

The components of the torsion object $S$ satisfy the differential comparisons modulo the basic forms $\omega^{\alpha}, \omega^{a}, \omega_{a}^{\alpha}$ :

$$
\begin{gathered}
\Delta S_{\beta \gamma}^{\alpha}-S_{[\beta a}^{\alpha} \omega_{\gamma]}^{a}+S_{[\beta \gamma]}^{\alpha a} \omega_{a} \equiv 0, \quad \Delta S_{\beta a}^{\alpha}-S_{\beta a}^{\alpha b} \omega_{b} \equiv 0, \quad \Delta S_{\beta \gamma}^{\alpha a}-S_{\gamma b}^{\alpha a} \omega_{\beta}^{b} \equiv 0, \\
\Delta S_{\beta b}^{\alpha a} \equiv 0, \quad \Delta S_{a \beta \gamma}^{\alpha}-S_{a[\beta b}^{\alpha} \omega_{\gamma]}^{b}+S_{a[\beta \gamma]}^{\alpha b} \omega_{b}-S_{\beta \gamma}^{\alpha} \omega_{a} \equiv 0, \quad \Delta S_{a \beta b}^{\alpha}-S_{a \beta b}^{\alpha c} \omega_{c}-S_{\beta b}^{\alpha} \omega_{a} \equiv 0, \\
\Delta S_{a \beta \gamma}^{\alpha b}-S_{a \gamma c}^{\alpha b} \omega_{\beta}^{c}+2 S_{a \beta \gamma}^{\alpha c b} \omega_{c}-S_{\beta \gamma}^{\alpha b} \omega_{a} \equiv 0, \quad \Delta S_{a \beta c}^{\alpha b}-S_{\beta c}^{\alpha b} \omega_{b} \equiv 0, \quad \Delta S_{a \beta \gamma}^{\alpha b c} \equiv 0, \\
\Delta S_{\alpha \beta}^{a}-S_{[\alpha b}^{a} \omega_{\beta]}^{b}+S_{[\alpha \beta]}^{a b} \omega_{b}+S_{\alpha \beta}^{\gamma} \omega_{\gamma}^{a} \equiv 0, \quad \Delta S_{\alpha b}^{a}+2 S_{b c}^{a} \omega_{\alpha}^{c}+S_{\alpha b}^{\beta} \omega_{\beta}^{a}-S_{b \alpha}^{a c} \omega_{c} \equiv 0, \\
\Delta S_{\alpha \beta}^{a b}-S_{c \beta}^{a b} \omega_{\alpha}^{c}+S_{\alpha \beta}^{\gamma b} \omega_{\gamma}^{a} \equiv 0, \quad \Delta S_{b c}^{a} \equiv 0, \quad \Delta S_{b \alpha}^{a c}+S_{\alpha b}^{\beta c} \omega_{\beta}^{a} \equiv 0 .
\end{gathered}
$$

From these comparisons we obtain the following theorem.

Theorem 4. The torsion object $S=\left\{S_{\beta b}^{\alpha a}=\delta_{\beta}^{\alpha} \delta_{b}^{a}, S_{\beta \gamma}^{\alpha}, S_{\beta a}^{\alpha}, S_{\beta \gamma}^{\alpha a}, S_{a \beta \gamma}^{\alpha}, S_{a \beta b}^{\alpha}\right.$, $\left.S_{a \beta \gamma}^{\alpha b}, S_{a \beta c}^{\alpha b}, S_{a \beta \gamma}^{\alpha b c}, S_{\alpha \beta}^{a}, S_{\alpha b}^{a}, S_{\alpha \beta}^{a b}, S_{b c}^{a}, S_{b \alpha}^{a c}\right\}$ of the connection $\Gamma$ is a tensor. It contains three elementary subtensors and four simple subtensors.

Remark 2. Since the torsion subobject $S_{\beta b}^{\alpha a}=\delta_{\beta}^{\alpha} \delta_{b}^{a}$ is a nonzero tensor, the connection $\Gamma$ is always a connection with torsion. 


\section{REFERENCES}

[1] O. Belova, "Connections in fiberings associated with the grassmann manifold and the space of centered planes," J. Math. Sci. (New York), vol. 162, no. 5, pp. 605-632, 2009, doi: 10.1007/s10958009-9649-y.

[2] J. Mike` s and et al., Differential geometry of special mappings. Palacký University Olomouc, Faculty of Science, Olomouc, 2015.

[3] E. G. Neifeld, "Affine connections on the normalized manifolds of planes in the projective space," News of High schools. Math., vol. 11, pp. 48-55, 1976.

[4] A. P. Norden, Spaces with an affine connection. Moscow: Nauka, 1976.

[5] Y. I. Shevchenko, Equipments of centreprojective manifolds, Kaliningrad, 2000.

Author's address

Olga Belova

Immanuel Kant Baltic Federal University, Institute of Physics, Mathematics and IT, 14 A. Nevskogo St., 236041 Kaliningrad, Russia

E-mail address: olgaobelova@mail.ru 